\title{
NONDISTRIBUTIVE RINGS AND THEIR ÖRE LOCALIZATIONS
}

\author{
MaŁgorzata ElżBieta Hryniewicka \\ Institute of Mathematics \\ University of Biatystok \\ Ciotkowskiego 1M, 15-245 Biatystok, Poland \\ e-mail: margitt@math.uwb.edu.pl
}

\begin{abstract}
In the paper, we introduce the notion of a nondistributive ring $N$ as a generalization of the notion of an associative ring with unit, in which the addition needs not be abelian and the distributive law is replaced by $n 0=0 n=0$ for every element $n$ of $N$. For a nondistributive ring $N$, we introduce the notion of a nondistributive ring of left quotients $S^{-1} N$ with respect to a multiplicatively closed set $S \subseteq N$, and determine necessary and sufficient conditions for the existence of $S^{-1} N$.

Keywords: semigroups, nearrings, nondistributive rings, nearrings of quotients, nondistributive rings of quotients, Öre localizations of nondistributive rings.
\end{abstract}

2010 Mathematics Subject Classification: 16Y99, 16Y30, 12K05, 16S85, $16 \mathrm{U} 20$.

\section{REFERENCES}

[1] K.L. Chew and G.H. Chan, On extensions of near-rings, Nanta Math. 5 (1971) $12-21$.

[2] J.R. Clay, Nearrings, geneses and applications (Oxford Science Publications, The Clarendon Press, Oxford University Press, New York, 1992).

[3] C.C. Ferrero and G. Ferrero, Nearrings, some developments linked to semigroups and groups (Advances in Mathematics (Dordrecht) 4, Kluwer Academic Publishers, Dordrecht, 2002).

[4] L.E. Dickson, Definitions of a group and a field by independent postulates, Trans. Amer. Math. Soc. 6 (1905) 198-204. doi:10.1090/S0002-9947-1905-1500706-2 
[5] D. Dorninger, H. Länger and M. Mạczyński, Ring-like structures with unique symmetric difference related to quantum logic, Discuss. Math. Gen. Algebra Appl. 21 (2001) 239-253. doi:10.7151/dmgaa.1041

[6] K. Głazek, A guide to the literature on semirings and their applications in mathematics and information sciences with complete bibliography (Kluwer Academic Publishers, Dordrecht, 2002).

[7] J.A. Graves and J.J. Malone, Embedding near domains, Bull. Austral. Math. Soc. 9 (1973) 33-42. doi:10.1017/S0004972700042830

[8] A. Hajnal and A. Kertész, Some new algebraic equivalences of the axiom of choice, Publ. Math. Debrecen 19 (1972) 339-340.

[9] M. Holcombe, Near-rings of quotients of endomorphism near-rings, Proc. Edinburgh Math. Soc., II Ser. 19 (1974/1975) 345-352. doi:10.1017/S0013091500010440

[10] T.Y. Lam, Lectures on modules and rings (Graduate Texts in Mathematics 189, Springer-Verlag, New York, 1999).

[11] S. Markov, On the algebra of intervals, Reliable Computing 21 (2016) 80-108.

[12] C.J. Maxson, On near-rings and near-ring modules (Doctoral dissertation, State University of New York at Buffalo, 1967).

[13] J.D.P. Meldrum, Near-rings and their links with groups (Research Notes in Mathematics 134, Pitman Advanced Publishing Program, Boston, 1985).

[14] O. Öre, Linear equations in non-commutative fields, Ann. Math., II Ser. 32 (1931) $463-477$. doi:10.2307/1968245

[15] A. Oswald, On near-rings of quotients, Proc. Edinburgh Math. Soc., II Ser. 22 (1979) 77-86. doi:10.1017/S0013091500016187

[16] G. Pilz, Near-rings, the theory and its applications, Second edition (North-Holland Mathematics Studies 23, North-Holland Publishing Co., Amsterdam, 1983).

[17] V. Seth, Near-rings of quotients (Doctoral dissertation, Indian Institute of Technology, 1974).

[18] V. Seth and K. Tewari, Classical near-rings of left and right quotients, Progr. Math. (Allahabad) 12 (1978) 115-123.

[19] M. Shafi, A note on a quotient near-ring, Arabian J. Sci. Engrg. 4 (1979) 59-62.

[20] H.S. Vandiver, Note on a simple type of algebra in which the cancellation law of addition does not hold, Bull. Amer. Math. Soc. 40 (1934) 914-920. doi:10.1090/S0002-9904-1934-06003-8 
[21] H.S. Vandiver, On the imbedding of one semi-group in another, with application to semi-rings, Amer. J. Math. 62 (1940) 72-78.

Received 10 October 2016 Revised 13 July 2017 Accepted 20 May 2018 